\title{
Synthesis and swelling characteristics of zwitterionic hydrogel
}

\author{
Meenakshi Singh, ${ }^{1 *}$ Paresh Kumar Singh, ${ }^{2}$ Vinay Kumar Singh ${ }^{2}$ \\ Department of Chemistry, Mahila MahaVidyalaya, Banaras Hindu University, \\ Varanasi-221005, India; e-mail: meenakshi_s4@rediffmail.com \\ 2 Department of Chemistry, U.P.A.College, Varanasi-221002, India.
}

(Received: 13 June, 2008; published: 27 December, 2008)

\begin{abstract}
Hydrogels comprising of zwitterionic repeat units were synthesized. The amine groups of the poly (ester amine) were quaternized with 1,3-propane sultone. The gelation was observed on treatment with sultone. The hydrophilic gel thus formed is sensitive to temperature, $\mathrm{pH}$, ionic strength and nature of electrolytes in aqueous solution. In absence of any electrolyte, the extent of swelling decreased with temperature, but in the monovalent salt solution the equilibrium swelling diminished with temperature and the contrary behaviour was experiential in bivalent electrolytes.
\end{abstract}

\section{Introduction}

Hydrogels, by definition, are three-dimensional cross-linked polymeric networks that can imbibe large amounts of water [1-4]. Based on the nature of cross-linking chemistry [5], this class of materials is categorized into physical gels consisting of polymeric networks bound together via polymer chain entanglement and/or noncovalent interactions that exist between polymeric chains [1, 3, 6, 7]. The forces accountable for the strength of such networks are characteristically based on hydrogen bonding, electrostatic or hydrophobic interactions and thus, the gels can be reversibly dissolved under certain conditions that would weaken these forces. Whereas, chemically crosslinked gels are bound together by covalent linkages, making them irreversible in the sense that the network structure cannot be ruined until the covalent bonds are destroyed.

The responsive properties make these materials promising in different fields including biotechnology, pharmaceutical, agriculture and industrial applications [8-11]. The possible applications include metal extraction, wastewater treatment [12], soft contact lenses, controlled release of drugs, enzyme supports, wound dressing, water managing materials, diapers, agrochemical release and others. Recently, a surge in the synthesis and characterization of synthetic polyampholytes has been reported [13-19]. Polyampholytic hydrogels are defined as crosslinked macromolecular networks containing positively and negatively charged repeating units and show very unusual properties from those of the parent polyelectrolyte polymers, which contain the same charge. Significant attention was given to synthetic polyampholytes due to their widespread applications as selective sorbents, membranes, flocculants, drug carriers etc. The synthetic polyampholytic gels bearing a resemblance to proteins incited them to use for the modeling of biological processes. In ampholyte hydrogels, 
the coulombic attraction between oppositely charged monomeric unit, in addition to the hydrogen bonding and hydrophobic interactions, play a crucial role to determine their phase transitions that leads to a different chain configuration within the network structures. The polyampholytic interpenetrating network hydrogel with the composition poly( $\mathrm{N}$-isopropylacrylamide-co-sodium acrylate)/ poly(ethyleneimine) was reported to be responsive to temperature, salts and with cationic surfactants as well [20]. Ogawa et al designed a biochemico-mechanical polyampholytic gel system consisting of $\mathrm{N}$-isopropylacrylamide (NIPAM), acrylic acid (AAc), and 1-vinylimidazole (VIM) units that undergo swelling and shrinking changes due to the electrostatic interaction between the opposite charges in the network [21]. Takeoka et al have described that polyampholytic gels undergo a discontinuous phase transition depending on the interactions between its repeating units in the gels [22]. Multiple phase transitions have been reported for methacryl-amido-propyl-trimethyl ammonium chloride (MAPTAC) and AAc hydrogels at different $\mathrm{pH}$ [23].

Because of the superior properties of polyampholytic hydrogels compared to those of the pristine polyelectrolytes and the specific utility in removing toxic heavy metal ions and organic compounds from sewage and industrial wastewater, we were interested to prepare the sulfobetaine gel (Poly-[N,N-methyl-bis-\{acetoethylene acetate\} piperazinium-bis-\{N-(3-sulfopropyl) ammonium betaine\}]). In this study, the zwitterionic sulfobetaine was synthesized and the swelling kinetics was studied in pure water, monovalent salt solution, divalent salt solution at varying temperatures and $\mathrm{pH}$.

\section{Results and Discussion}

Zwitterionic gels have both positively and negatively charged groups. The polymeric gels consisting of zwitterionic monomers have electrostatic attractive forces between the two opposite charges, which keep them in collapsed state. These gels can be made by either having oppositely charged groups on the polymer backbone, commonly termed as polyampholytes or on the same monomer unit known as polybetaines, due to ubiquitous use of betaine monomers [25, 26].

In this study, the alkylsulfonate group imparts negative charge, while the positive charge is introduced by the quaternary ammonium centers. The sulfoalkylbetaine groups have $\mathrm{pH}$ independent zero net charge. The coulombic attraction between the opposite charges provides the cross-links needed for gelation. Cross-linker from outside was not required here.

\section{Effect of swelling time}

Figure 1 shows the swelling behaviour in deionized water. The extent of swelling was found to be largest in first 45 minutes. In approximately 45 minutes, the equilibrium swelling state was achieved. Transparent gel samples were swollen uniformly and had smooth edges and compact shape.

\section{Effect of ionic strength}

The extent of swelling is dictated by the composition of swelling medium. Addition of low mol.wt electrolyte has tremendous effect on the swelling behaviour of this gel (Figure 2). The presence of monovalent salts deswells the polymeric betaines. Addition of $\mathrm{KCl}$ or $\mathrm{NaCl}$ provides competition for the water molecules hydrating the polybetaines chains. Dehydration of the polybetaine chain results in deswelling. The 
overall swelling is the net effect of that due to salt imbibed within the gel (which increases continuously with time) and that due to water taken into the gel. The quaternary ammonium and sulphonate ions are surrounded by a sheath of $\mathrm{Cl}^{-}$and $\mathrm{K}^{+}$ ions (in $\mathrm{KCl}$ solution) respectively. Hence, zwitterionic character is retained.

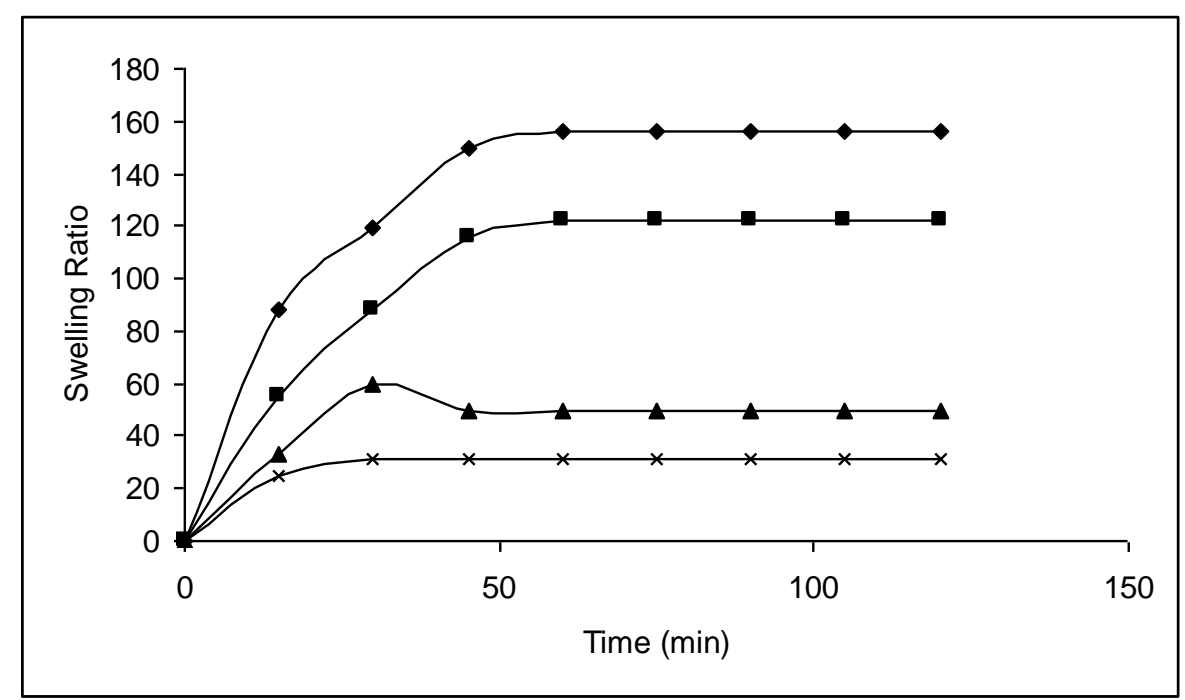

Fig. 1. Swelling behaviour of zwitterionic hydrogel in water at $20{ }^{\circ} \mathrm{C}(\bullet), 30^{\circ} \mathrm{C}(\cdot), 40$ ${ }^{\circ} \mathrm{C}(\mathbf{\Delta})$ and $50{ }^{\circ} \mathrm{C}(\mathrm{x})$.

The possibility of strong intra-chain or inter-chain interactions is restored, i.e. breakage of such interactions to allow ingress of water is reduced. Consequently, the overall swelling decreases at high concentration of salt. Here, four different electrolytes having common anion $\left(\mathrm{Cl}^{-}\right), \mathrm{NaCl}, \mathrm{KCl}, \mathrm{MgCl}_{2}$ and $\mathrm{CaCl}_{2}$ (Figure 2) were analyzed.

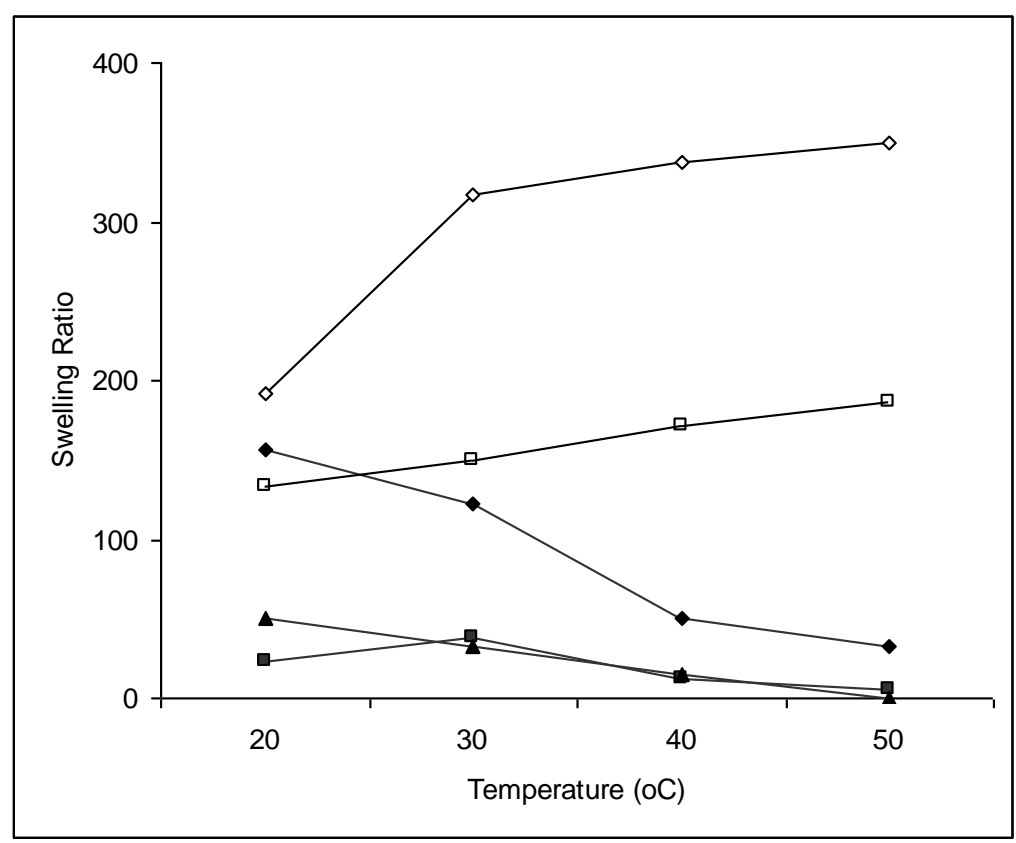

Fig. 2. Swelling behaviour of zwitterionic hydrogel in water ( $\bullet$, in $\mathrm{NaCl}(\mathbf{\Delta})$, in $\mathrm{KCl}(\cdot)$, in $\mathrm{MgCl}_{2}(\diamond)$ and in $\mathrm{CaCl}_{2}(\square)$ at various temperatures. 
With respect to temperature, the swellability decreases in the presence of monovalent salt solutions, i.e., $\mathrm{NaCl}$ and $\mathrm{KCl}$. The extent of swelling was more in the presence of $\mathrm{NaCl}$ than in $\mathrm{KCl}$. Disruption and desolvation of the $\left(>\mathrm{C}=\mathrm{O},-\mathrm{SO}_{3}{ }^{-}\right)-\mathrm{H}_{2} \mathrm{O}$ hydrogen bonds allows attractive interchain hydrophobic interactions and hydrogen bonding to dominate. Thus the gel deswells and contracts. Whereas, in the salt solutions of the divalent electrolytes, the swellability enhances in comparison to that in pure deionized water (Figure 2). The divalent cations are more effective in curtailing the charge centers, thus enhancing the water uptake by hydrophilic groups of the polymer chain. The divalent ions facilitated the disruption of crosslinks formed between $-\mathrm{SO}_{3}$ of one chain with cationic centre of another chain by electrostatically binding two of the sulfoalkyl betaine groups of a chain simultaneously. This disruption of crosslinks between chains assists in better swelling. Here, four different electrolytes having common anion $\left(\mathrm{Cl}^{-}\right), \mathrm{NaCl}, \mathrm{KCl}, \mathrm{MgCl}_{2}$ and $\mathrm{CaCl}_{2}$ have been deliberated (Figure 2). Among them, $\mathrm{K}^{+}$has least charge density and $\mathrm{Mg}^{2+}$ has most. The presence of $\mathrm{Na}^{+}$and $\mathrm{K}^{+}$ions deswell the polymeric gel, being incapable of breaking strong attractive forces between the permanent charges of the polymeric backbone. The electrostrictional effect is curtailed encouraging the solvophobicity of the polymeric chain providing pockets to accommodate more and more water molecules. Moreover, the smaller hydration sphere of bigger cations is able to penetrate the gel crosslinks whereas the smaller ions $\left(\mathrm{Na}^{+}, \mathrm{K}^{+}\right)$with larger hydration spheres were unable to penetrate the gel network, thus deswelling the gel.

The thermosensitivity is also reversed in the presence of divalent salts. The increase in temperature enhances the swellability as shown by class (c) as grouped in subsequent section.

\section{Effect of $\mathrm{pH}$}

Such ionic gels are $\mathrm{pH}$ responsive also. At higher temperature, the acidic $\mathrm{pH}$ increases the swelling, whereas at higher $\mathrm{pH}$, the gel deswells (Figure 3,4 ).

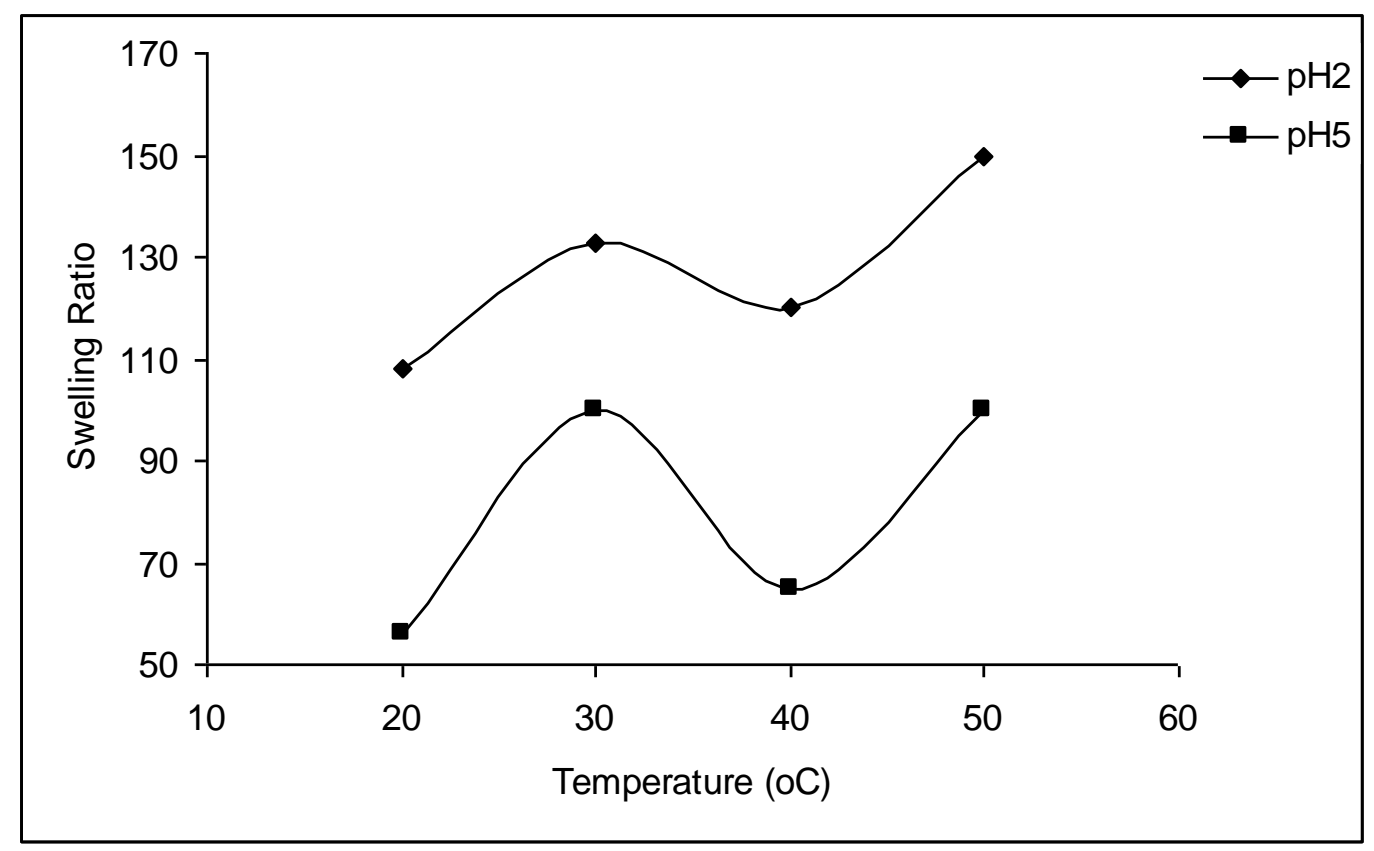

Fig. 3. Swelling behaviour of zwitterionic hydrogel in water at $\mathrm{pH} 2(\bullet), \mathrm{pH} 5(\cdot)$ at various temperatures. 
Although sulfoalkyl betaine groups has $\mathrm{pH}$ independent zero net charge, in the presence of $\mathrm{H}^{+}$(particularly at $\mathrm{pH}<\mathrm{pK}_{\mathrm{a}}$ ), the ionic crosslinks between the chains are disrupted permitting more and more swelling of the network, while at $\mathrm{pH}>\mathrm{pK}_{\mathrm{a}}$, the sulfoalkyl betaine groups remain ionized, re-forming the ionic crosslinks between the chains and keeping the network in collapsed state. The $\mathrm{H}^{+}$ions at lower $\mathrm{pH}$ (below $\mathrm{pK}_{\mathrm{a}}$ ) block the $\mathrm{SO}_{3}{ }^{-}$charge centers to break the interionic coulombic attractions, thus enhancing more and more ingress of water. At the lower $\mathrm{pH}$, with high concentration of $\mathrm{H}^{+}$, the ionic interaction between $\mathrm{SO}_{3}{ }^{-}$and $\mathrm{N}^{+}$is broken, while at higher $\mathrm{pH}$, the acidic sites remain ionized thus again making the ionic cross links to collapse the gel network. At low $\mathrm{pH}$, the extent of swelling after an initial dip augments with temperature rise.

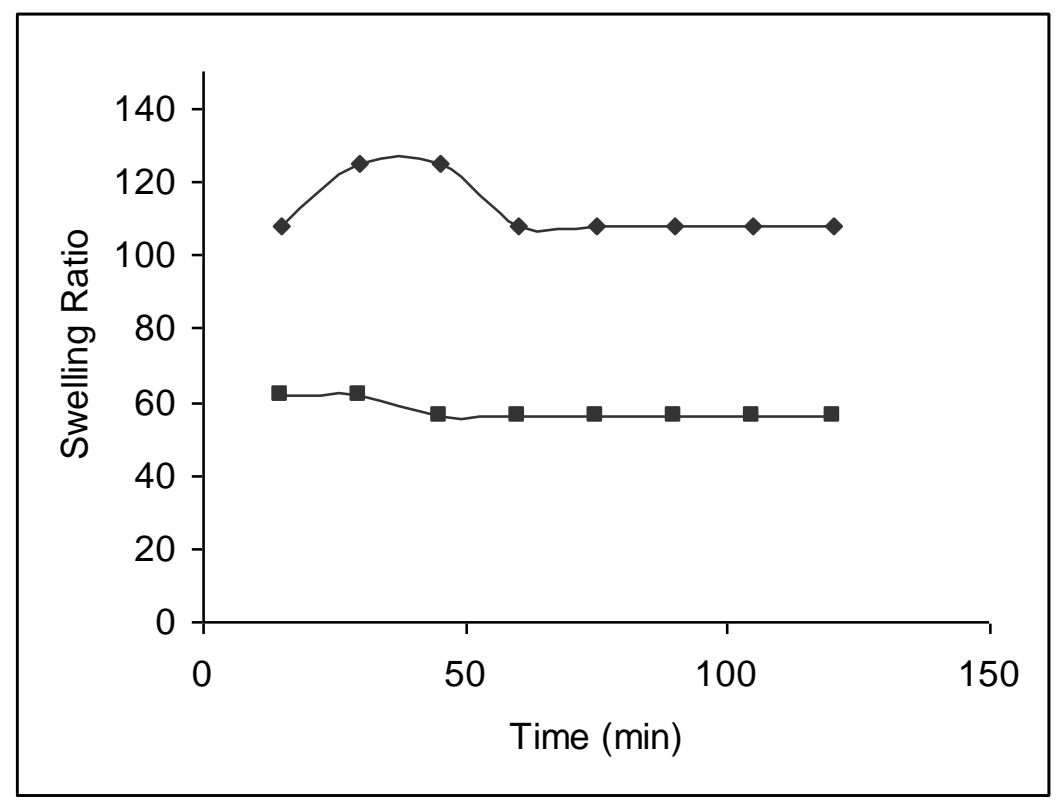

Fig. 4. Swelling behaviour of zwitterionic hydrogel in water at $\mathrm{pH} 2(\triangleleft), \mathrm{pH} 5(\cdot)$ at $20^{\circ} \mathrm{C}$.

\section{Effect of temperature}

The swelling ratio decreased with temperature from 20 to $50^{\circ} \mathrm{C}$ as shown in Figure 2 . The comparison with other sulfobetaine hydrogels will be speculative as among the rather few systems studied, they differ in nature and content of crosslinker. As categorized elsewhere, the effect of temperature on swelling of hydrogels may be (a) swelling decreases with temperature [27-33] or (b) a combined effect of swelling decreasing with temperature down to a minimum value and thereafter increasing at higher temperature $[28,29\}$.or (c) swelling increases with temperature or (d) an unusual additional case is a hydrogel which undergoes an increase in swelling with temperature up to a maximum and thereafter a decrease in swelling [34]. In this sample, the swelling diminished uniformly with temperature as in class (a). Another significant observation was that the gel showed a swelling plateau at higher temperatures.

\section{Conclusions}

A 'responsive' zwitterionic polyelectrolytic gel is synthesized, showing sensitivity to temperature, $\mathrm{pH}$ and ionic strength. The gel network attains equilibrium swelling 
rapidly. The divalent ions were able to disrupt the ionic crosslinks more effectively than the monovalent ions. This sensitivity towards low mol wt electrolytes affects thermal sensitivity as well in similar manner. Such sensitivity could be exploited in drug delivery and other such applications. Such responsive gels have significant contribution in controlled delivery.

\section{Experimental}

\section{Synthesis}

Poly-[N,N-methyl-bis-\{acetoethylene acetate $\}$ piperazinium-bis-\{N-(3-sulfopropyl) ammonium betaine] : $20 \mathrm{mmol}$ 1-(2-aminoethyl)-piperazine(AP) $(2.58 \mathrm{~g}$ in $15 \mathrm{~mL}$ DMF) was added to $20 \mathrm{mmol}$ poly (ethylene glycol) diacrylate(PEODA) $(14 \mathrm{~g}$ in $15 \mathrm{~mL}$ DMF) and kept at room temperature for 8 days. Subsequently, $20 \mathrm{mmol}$ of propane1,3-sultone (2.44g in $15 \mathrm{~mL}$ DMF) was added and kept at room temperature for 4 days. A hard solid light yellow coloured gel was recovered. The gel was washed several times with water and dried at $50{ }^{\circ} \mathrm{C}$ until constant weight. Analysis (\%) found: $\mathrm{C}=46.1, \mathrm{H}=6.5 ., \mathrm{N}=4.0$; calculated: $\mathrm{C}=45.4, \mathrm{H}=7.1, \mathrm{~N}=4.7$. Thus hydrogels comprising of zwitterionic repeat units were synthesized (Figure 5).

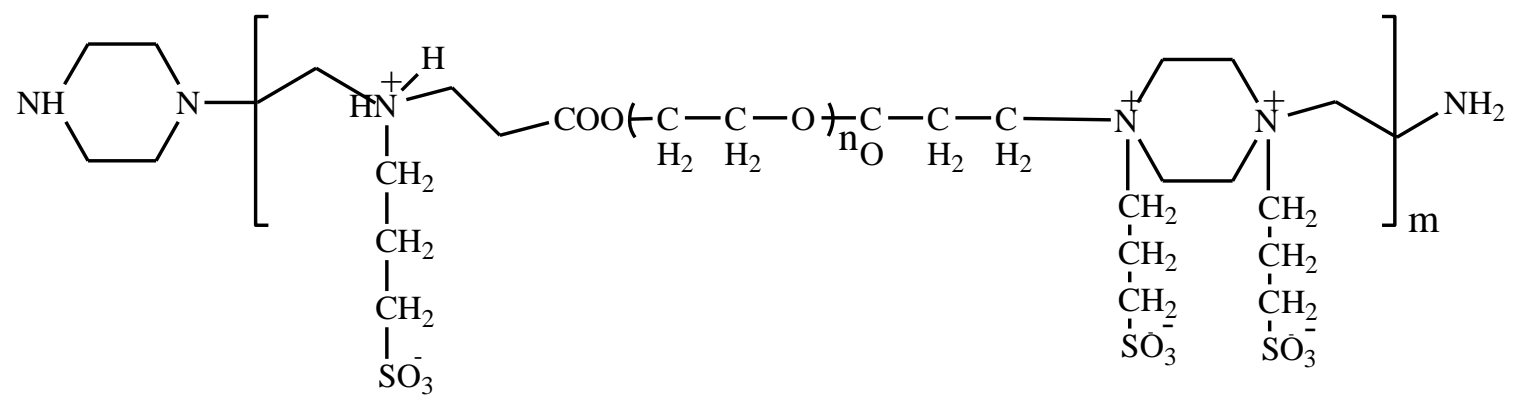

Fig. 5. Chemical structure of zwitterionic polymer.

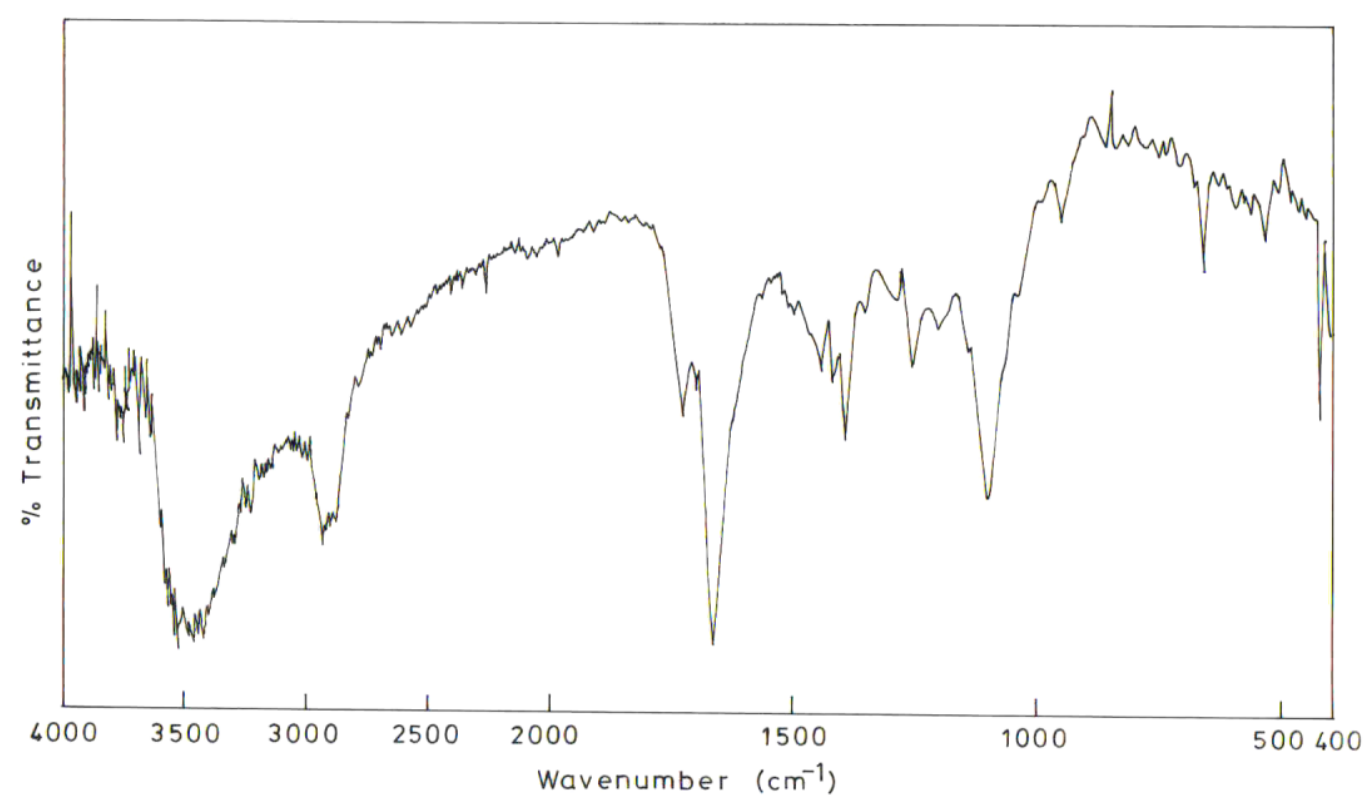

Fig. 6. IR spectra of zwitterionic polymer. 
The diamine 1-(2-aminoethyl) piperazine (AP) was added to poly(ethyl glycol) diacrylate in a 1:1 ratio via Michael addition to form the poly(ester amine) as reported elsewhere [24]. The secondary amino group of diamine reacts faster with the vinyl group of diacrylate (nucleophilic addition). The amine groups of the poly(ester amine) were quaternized with 1,3-propane sultone. The gelation was observed on treatment with sultone.

The compound exhibits characteristic IR $(\mathrm{KBr})$ absorption bands (Figure 6) at 2930 $\mathrm{cm}^{-1}$ (C-H asymmetric stretching) along with the absorption at $1440 \mathrm{~cm}^{-1}$ due to asymmetric bending of $\mathrm{C}-\mathrm{H}$ bond and the symmetric bending at $1390 \mathrm{~cm}^{-1}$. The characteristic absorption for sulfonate groups appear at $1201 \mathrm{~cm}^{-1}$ and $1099 \mathrm{~cm}^{-1}$. The strong absorption at $1664 \mathrm{~cm}^{-1}$ corresponds to $>\mathrm{C}=\mathrm{O}$ group of poly(ethylene glycol)acrylate. The peaks at $3300 \mathrm{~cm}^{-1}$ (N-H stretching), $1253 \mathrm{~cm}^{-1}$ (C-N stretching) correspond to the1-(2-aminoethyl)-piperazine(AP).

\section{Swelling Measurements}

Samples of known weight were immersed in excess of distilled water and kept in a sealed container, which were placed in temperature-control bath with $\pm 1^{\circ} \mathrm{C}$ accuracy until equilibrium was reached. The samples were removed and blotted dry. The swelling ratio of the copolymer gels was determined gravimetrically. The equilibrium weight of the swollen samples at each temperature was determined. The swelling ratios of the copolymer gels were calculated from the ratio of the weight of the equilibrated gel to the dry weight

Swelling Ratio $=\left(W_{s}-W_{d} W_{d}\right) \times 100$

where $W_{s}=$ Weight of the swollen gel, $W_{d}=$ Weight of the dry gel sample.

\section{References}

[1] Hoffman, A.S. Adv. Drug Delivery Rev., 2002, 54, 3.

[2] Drury, J.L.; Mooney, D.J. Biomaterials 2003, 24, 4337.

[3] DeRossi, D.; Kajiwara, K.; Osada, Y.; Yamauchi, A. Polymer Gels: Fundamentals and Biomedical Applications, Plenum Press: New York, 1991.

[4] Dhara, D.; Nisha, C.K.; Chatterji, P.R. Macromol. Sci., Pure Appl. Chem., 1999, A36, 197.

[5] Hennink, W.E. Adv. Drug Delivery Rev., 2002, 54,13.

[6] Akiyoshi, K.; Kang, E.C.; Kurumada, S.; Sunamoto, J.; Principi, T.; Winnik, F.M. Macromolecules, 2000, 33, 3244.

[7] Collier, J.H.; Hu, B.H.; Ruberti, J.W.; Zhang, J.; Shum, P.; Thompson, D.H.; Messersmith, P.B. J. Am. Chem. Soc., 2001, 1239463.

[8] Schlid, H.G. Prog. Polym. Sci., 1992, 17,163.

[9] Tanaka, Y.; Gong, J.P.; Osada, Y. Prog. Polym. Sci., 2005, 30, 1.

[10] Gil, E.S.; Hudson, S.M. Prog. Polym. Sci., 2004, 29, 1173.

[11] Osada, Y.; Gong, J.P.; Tanaka, Y. J.Macromol. Sci.Part C: Polym Rev., 2004, $44,87$.

[12] Paulino, A.T.; Guilherme, M.R.; Reis, A.V.; Campese, G.M.; Muniz, E.C.; Nozaki, J. J.Colloid Interface Sci., 2006, 301, 55.

[13] Ali, S.A.; Ali,A. Polymer, 2001, 42, 7961.

[14] Hadjikallis, G.; Hadjiyannakou, S.C.; Vamvakaki, M.; Patrickios, C.S. Polymer, 2002, 43, 7269. 
[15] Sutani, K.; Kaetsu,I.; Uchida,K.; Matsubara,Y. Radiat.Phys. Chem., 2002, 64, 331.

[16] Kakinoki, S.; Kaetsu, I.; Nakayama, M.; Sutani, K.; Uchida, K.; Yukutake, K. Radiat.Phys. Chem., 2003, 67, 685.

[17] Koizhaiganova, R.B.; Kudaibergenov, S.E.; Geckeler, K.E. Macromol. Rapid Commun., 2002, 23, 1041.

[18] Noh,J.G.; Sung,Y.J.; Geckeler, K.E.; Kudaibergenov,S.E. Polymer, 2005, 46, 2183.

[19] Xue, W.; Huglin, M.B.; Liao, B. European Polym. J., 2006, 42, 3015.

[20] Murali Mohan, Y.; Geckeler, K.E. Reactive and Functional Polymers, 2007, 67, 144.

[21] Ogawa, Y.; Ogawa, K.; Wang, B.; Kokufuta, E. Langmuir, 2001, 17, 2670.

[22] Takeoka, Y.; Berker, A.N.; Du, R.; Enoki, T.; Grosberg, A.Y.; Kardar, M.; Oya, T.; Tanaka, K. ; Wang, G.; Yu, X.; Tanaka, T. Phys.Rev.Lett., 1999, 82, 4863.

[23] Mafe, S.; Manzanares, J.A.; English, A.E.; Tanaka, T. Phys.Rev.Lett., 1997, 79, 3086.

[24] Gao, C.; Tang, W.; Yan, D. J.Polym.Sci.:Polym.Chem., 2002, 40, 2340.

[25] Lowe, A.B.; McCormick, C.L. Chem. Rev., 2002, 102, 4177.

[26] Singh, P.K.; Singh, V.K. Singh, M. e-Polymers, 2007, 30, 1.

[27] Huglin, M.B.; .Zakaria, M.B.; Rehab, M.A. Macromolecules, 1986, 19, 2986.

[28] Refojo, M.F. J Appl Polym Sci., 1966, 9, 3161.

[29] Dušek, K.; Bohdanecky, M.; Propokova, E. Eur Polym J., 1974, 10, 239.

[30] Dušek, K.; Bohdanecky, M.; Vosicky, V. Collect Czech Chem Commun., 1977, 42, 1599.

[31] Warren, T.C.; Prins,W. Macromolecules, 1972, 5, 506.

[32] Peppas, N.A.; Merrill, E.W. J Polym Sci, Polym Chem Ed, 1976, 14, 459.

[33] Sakurada, I.; Nakajima, A.; Fujiwara, H. J Polym Sci, 1959, 35, 497.

[34] Huglin, M.B.; Radwan, M.A. Polymer, 1991, 32, 3381. 